\title{
Time-resolved PIV measurements of a deflected submerged jet interacting with liquid-gas and liquid-liquid interfaces
}

\author{
Stefan Puttinger ( $\varangle)$, Mahdi Saeedipour \\ Department of Particulate Flow Modelling, Johannes Kepler University, 4040 Linz, Austria
}

\begin{abstract}
This paper presents an experimental investigation on the interactions of a deflected submerged jet into a liquid pool with its above interface in the absence and presence of an additional lighter liquid. Whereas the former is a free surface flow, the latter mimics a situation of two stratified liquids where the liquid-liquid interface is disturbed by large-scale motions in the liquid pool. Such configurations are encountered in various industrial applications and, in most cases, it is of major interest to avoid the entrainment of droplets from the lighter liquid into the main flow. Therefore, it is important to understand the fluid dynamics in such configurations and to analyze the differences between the cases with and without the additional liquid layer. To study this problem, we applied time-resolved particle image velocimetry experiments with high spatial resolution. A detailed data analysis of a small layer beneath the interface shows that although the presence of an additional liquid layer stabilizes the oscillations of the submerged jet significantly, the amount of kinetic energy, enstrophy, and velocity fluctuations concentrated in the proximity of the interface is higher when the oil layer is present. In addition, we analyze the energy distribution across the eigenmodes of a proper orthogonal distribution and the distribution of strain and vortex dominated regions. As the main objective of this study, these highresolution time-resolved experimental data provide a validation platform for the development of new models in the context of the volume of fluid-based large eddy simulation of turbulent two-phase flows.
\end{abstract}

\author{
Keywords \\ submerged jet \\ liquid-liquid interface \\ time-resolved PIV \\ LES validation \\ continuous casting mold flow \\ Article History \\ Received: 17 September 2020 \\ Revised: 29 October 2020 \\ Accepted: 28 December 2020 \\ Research Article \\ (c) The Author(s) 2021
}

\section{Introduction and motivation}

\subsection{Problem definition}

The term submerged jet is widely used and appears in various contexts for single- or multiphase flow problems. Thus, we first need to define the scope of this work. A submerged jet in general can be any kind of gas, liquid, or solid particle jet in an environment of the same or different type of material. The simplest case, of course, is a liquid jet of water injected in a stagnant water pool or a gas jet released into ambient air at rest. The flow field and turbulence characteristics of such jets can usually be measured by different experimental techniques such as particle image velocimetry (PIV) (Raffel et al., 2007; Adrian and Westerweel, 2011), hot wire anemometry (CTA) (Bruun, 1995), or laser doppler anemometry (LDA) (Albrecht et al., 2003).

Gas jets in water are driven by inertia and buoyancy

$\triangle$ stefan.puttinger@jku.at and have many applications in hydraulic engineering, oceanography, or metallurgical flows. As a consequence of the bubble formation, they are less accessible for quantitative methods like PIV or LDA. Hence, they are mainly tackled by image processing to obtain at least global features of the flow (Weiland and Vlachos, 2013; Harby et al., 2014; Berna et al., 2017; Ma et al., 2018; Dong et al., 2020). Likewise, it is not possible to measure jet velocities in highly laden particle jets commonly used for the burning of solid fuels (Birzer et al., 2009, 2011, 2012; Puttinger et al., 2013).

The velocity field and turbulence characteristics of single phase unbounded jets are well documented in literature (e.g., Wygnanski and Fiedler, 1969). Indeed, most technical applications are bounded flows where the jet interacts with walls and/or interfaces. Depending on the distances to the walls and interfaces the flow profiles may differ significantly compared to a free jet.

In this study, we will focus on a water jet released into a 


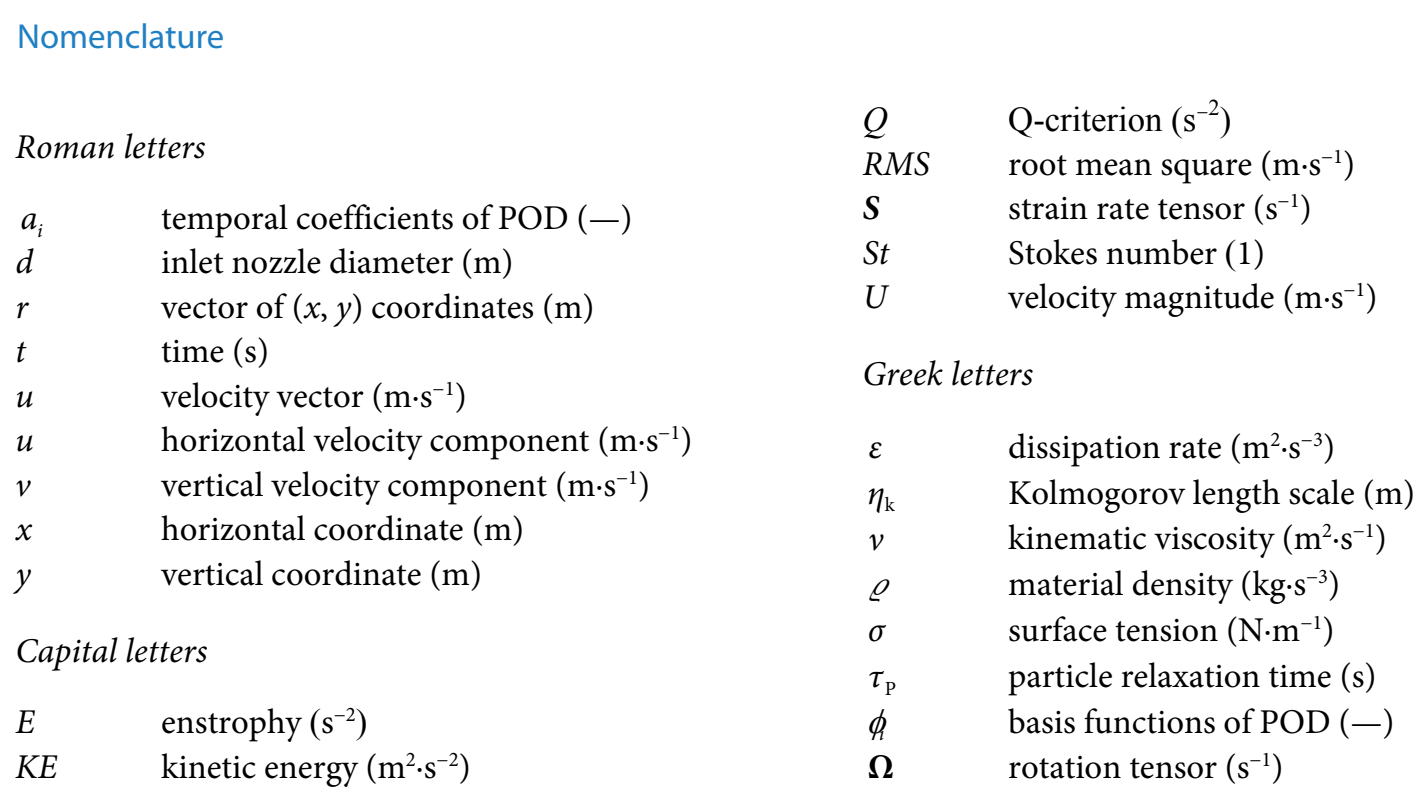

basin of water with a constant fill level and discuss the interaction of the jet with a free surface or an additional oil layer. Although the jet itself is a single-phase problem (jet of water in a water pool), in the remainder of this paper the jet interacting with the free surface is called the two-phase problem and the jet interacting with the liquid-liquid and air interfaces is referred to as the three-phase problem.

\subsection{Technical background and application}

Our starting point to discuss the technical background for such a flow situation originates from steel industry despite its vast application in other industries. A continuous casting mold essentially consists of the same setup. A liquid jet of steel is released from the exit ports on both sides of a submerged entry nozzle (SEN). The jets are then deflected up- and downwards on the outer walls of the casting mold and the upward deflected parts of the jet interact with the steel-slag interface (Fig. 1). The exit ports usually have an orientation in the range 0 to $-20^{\circ}$ downwards.

The avoidance of slag entrainment and non-metallic inclusions in the final product is the major goal in continuous casting. Hibbeler and Thomas (2013) discussed various reasons for slag entrainment. The three major mechanisms are

(1) vortex formation in the proximity of the SEN due to asymmetries in the global flow,

(2) interaction or argon bubbles with the slag layer,

(3) high shear forces at the steel-slag interface due to the upward deflected part of the jet.

It is well known from literature that the jets exiting the SEN are not stable but tend to oscillate up and down in the

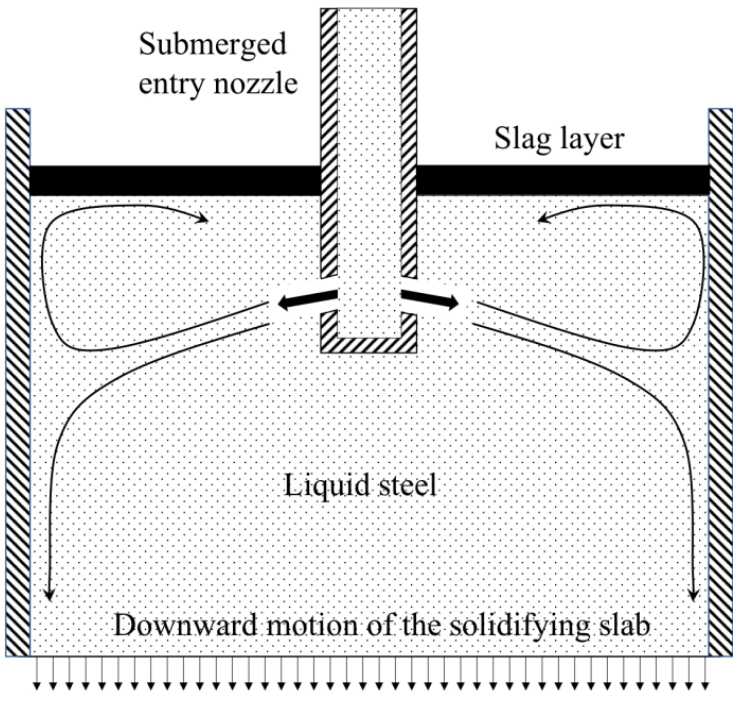

Fig. 1 Outline of a continuous casting mold in steel slab production.

mold. Gebert et al. (1998) and Zhang and Shen (2006) have shown this behavior numerically for a single vertically oriented jet. Lawson and Davidson (2001) and Lawson et al. (2005) reported PIV and LDA experiments on a similar setup. Besides an inherent instability of the flow, asymmetries can be triggered by clogging of the SEN (Srinivas et al., 2019) and also the bended geometry of the mold downward of the pure liquid zone might have an influence on the flow symmetry (Liu and Li, 2018). Consequently, if there is a net horizontal mean flow around the SEN caused by such asymmetries, vortices will occasionally form on the leeward side of the nozzle that possibly entrain slag into the mold (Pirker, 2010). 
The usage of argon injection can reduce the attachment of non-metallic inclusions to the inner walls of the SEN, and thus, reduce SEN clogging. In addition, the argon bubbles can capture these inclusions and transport them to the slag layer. However, the interaction of a large number of argon bubbles with the steel-slag interface can have a contrary effect and enforce slag entrainment. Yu et al. (2010) presented a numerical study on the interface motion due to argon injection. Ramos-Banderas et al. (2005) and Liu et al. (2014) conducted numerical and experimental studies on mold flow with argon bubbles.

In the present work we will focus on the third mechanism in the above list, namely shear layer instabilities. We do not use additional gas injection in our experiments and we eliminate flow asymmetries by replacing the SEN with a symmetry plane as discussed in detail in Section 2. The primary goal of the presented experiments is to provide high-quality validation data for computational fluid dynamics (CFD) model development.

\subsection{State of the art in numerical modelling}

The highly unsteady and turbulent flow conditions in the continuous casting mold demand a certain level of accuracy in turbulence modelling to analyze and control local phenomena such as slag entrainment and mass transfer across the interface. The typical CFD approach to study such complex flow consists of solving Navier-Stokes equations with proper turbulence models in combination with an interface capturing technique such as the VOF method (Hirt and Nichols, 1981). Despite their traditional affordability for industrial applications, Reynolds-averaged NavierStokes-based turbulence models commonly fail to picture multiphase turbulence. The advances in computational tools during the past decade have made the large eddy simulation (LES) affordable for the investigation of multiphase flows. Many authors have employed LES to tackle the continuous casting mold flow problem (Pirker, 2010; Ji et al., 2013; Khan et al., 2018; Liu and Li, 2018). Nevertheless, the LES of multiphase flows suffers from major shortcomings yet to be overcome. Most of these studies follow the conventional LES concept which is basically the single-phase LES formalism where only subgrid-scale (SGS) stresses are modelled using an eddy-viscosity approach, and neglect other SGS physics such as surface tension and interface dynamics.

Although different studies in the literature (Larocque et al., 2010; Vincent et al., 2018) have underlined the importance of all these SGS terms for the development of interfacial physics, their modelling and analysis have remained limited to some recent contributions. Ketterl and Klein (2018), Klein et al. (2019), and Hasslberger et al. (2020) have conducted comprehensive a-priori assessments on all the two-phase flow SGS terms based on the volume of fluid (VOF) method. They analyzed different existing subgrid closure models among which the scale-similarity concept shows a convincing performance. Vincent et al. (2018) conducted an a-priori analysis based on a turbulent phase inversion problem and reported that the approximate deconvolution method (ADM) (Stolz and Adams, 1999) reveals the highest correlations with data from direct numerical simulations (DNS), and therefore, is a better candidate for modelling all the SGS terms in the two-phase LES context. Consequently, Saeedipour et al. (2019b) developed the ADM-VOF approach for the a-posteriori LES of two-phase flows, which then was employed to simulate the continuous casting mold flow with a better prediction of the macroscopic interfacial features compared to conventional LES (Saeedipour et al., 2019a). Recently, Saeedipour and Schneiderbauer (2019) have introduced a fully functional approach for the two-phase LES that includes surface tension effects into the subgrid eddy-viscosity and has revealed a-priori competence for the accurate VOF simulation of interfacial flows.

Clearly, a general conclusion on the most accurate approach is still elusive and closure model development for multiphase LES is an ongoing research topic that, in turn, demands high-fidelity data for validation purposes. Nevertheless, most of the aforementioned works have done the model validations against highly-resolved simulation data, which is hard to achieve in the case of real-scale applications. This explains the importance of experimental benchmarking and time-resolved data acquisition for turbulent liquidliquid and liquid-gas flows, which is the major objective of the present study.

\subsection{Previous experimental works}

There are a large number of experimental studies on jets (Wygnanski and Fiedler, 1969; Westerweel et al., 2009), jets in cross-flow (Meyer et al., 2007; Karagozian, 2014; Altaharwah et al., 2020), or impinging jets (Fitzgerald and Garimella, 1998; Guo et al., 2017). While in the first two cases the turbulent interaction of the jet with its surrounding fluid is of major interest, the typical application of the latter case is heat transfer. Measurements on submerged jets were reported by several authors. Kwon and Seo (2005) were focusing on the averaged flow profiles of jets at different Reynolds numbers. Lacagnina and Romano (2015) investigated the influence of the optical magnification on the flow statistics of a submerged jet. Ganapathisubramani et al. $(2007,2008)$ used stereo PIV to measure the flow characteristics of submerged jet. Time-resolved data of a turbulent jet was reported by Cheng et al. (2011) and Semeraro et al. (2012). Wen et al. (2014) conducted experiments on a submerged 
jet interacting with a free surface.

Concerning the more specific situation of the continuous casting mold flow, the conventional flow characterization techniques would be impractical due to the high-temperature conditions in the real process. Thus, it has been common in the literature to use an analogous water-oil experimental setup where water and oil resemble the molten steel and slag layer, respectively. However, the majority of older publications neglected the oil layer and conducted pure water experiments (Yokoya et al., 2000; Zhang et al., 2007; Ren et al., 2019) or focused on the effect of gas injection (Ramos-Banderas et al., 2005; Liu et al., 2014).

Vakhrushev et al. (2014), Zhao et al. (2017), and Zhao and Zhou (2019) report experiments for LES validation with a water and oil configuration but focus on the analysis of the interface deformation and open eye formation without further measurements of the flow field. Campos et al. (2015) conducted point wise measurements at various distances to the liquid-liquid interface via LDA. André and Bardet (2015) extracted the interfacial shear stress of a free surface from simultaneous PIV measurements in the gas and liquid phase.

\subsection{Novelty of this study}

As mentioned above, the major goal of this work is to provide high-quality data to support LES modelling activities for two- and three-phase flows. Of course, it would be nice to obtain the shear stresses of the water-air and water-oil-air interfaces. However, due to the highly unsteady threedimensional motion of a liquid-gas and liquid-liquid interface, part of the near-interface area can be temporarily hidden when using a standard PIV setup with a $90^{\circ}$ camera angle. Since the interface deformation causes heavy light reflections of the laser light sheet, the usage of oblique camera angles suffers from high data loss and cannot entirely solve this problem. Hence, it is not possible to fully resolve the interface region in a sufficient quality to reliably extract interfacial shear stresses. Using a similar open channel setup like André and Bardet (2015) might be more successful in this regard. However, the authors decided to stick with the mold flow setup and use standard planar PIV measurements $(2 \mathrm{D}-2 \mathrm{C})$ at a $90^{\circ}$ angle but focus on a high temporal and spatial resolution. Since the spatial filtering nature of LES is separating the energy cascade into resolved and unresolved parts, the energy content of the latter should be captured correctly by an adequate subgrid model. As a consequence, there is a need for highly resolved validation data for two- and three-phase flows. In contrast to the usual approach to use DNS data for LES validation, this study presents a detailed flow analysis that is purely based on highly resolved experimental PIV data. The presented data herein allows us to analyze the energy distribution close to the interface even though the interface itself might not be fully resolved.

\section{Experimental setup and data processing}

\subsection{Apparatus}

The experimental setup was inspired by the geometry of a continuous casting mold in steel industry. In a real casting mold, the liquid steel is poured into the mold from the exit ports of a submerged entry nozzle. The SEN is usually a simple cylindrical pipe but the precise geometry of the exit ports can vary strongly with the application and type of steel grading. The experimental apparatus depicted in Fig. 2 is a simplified setup of such a continuous casting mold. The SEN is simply replaced by a symmetry plane with a circular inlet nozzle. The angle of the inlet angle can be adjusted in a wide range from $-30^{\circ}$ (downward jet direction) to $+10^{\circ}$ (upward jet direction). Thus, also extreme cases can be captured with this setup without having to build multiple SENs. However, the data presented in this paper only covers the simplest and most general case of $0^{\circ}$ inlet angle. One half of the basin from the side wall to the symmetry plane is $250 \mathrm{~mm}$. The fill level from the porous plate to the free water surface is $315 \mathrm{~mm}$. For the test cases with additional oil layer its thickness was set to $15 \mathrm{~mm}$. The width of the basin is $70 \mathrm{~mm}$. These dimensions roughly correspond to a 1:3 scaling of a real continuous casting mold where a typical slab width is $1500 \mathrm{~mm}$. The inlet nozzle diameter $d=20 \mathrm{~mm}$.

The flow rate of the pump was 0.265 liters per minute, resulting in an exit velocity of $u_{\text {jet }}=0.85 \mathrm{~m} / \mathrm{s}$ and a Reynolds number based on the nozzle diameter of $R e_{\text {jet }}=16870$. For continuous casting the relevant numbers are the mass flow rate in tons per min and the slab speed at which it is leaving the mold in $\mathrm{m} / \mathrm{min}$. With the given numbers of our experiment the casting speed results in $0.92 \mathrm{~m} / \mathrm{min}$ which approximately hits the average values of casting speeds which are in a typical range of $0.5-1.5 \mathrm{~m} / \mathrm{min}$. Certainly, achieving physical similarity in all relevant numbers and material properties is almost impossible for modelling steel and slag flows because of the large density differences. In the present experiments, paraffin oil is used as the lighter liquid to mimic the slag layer on top of the main water flow. It is clear from Table 1 that the difference in density between water and paraffin oil is much lower than between steel and slag. However, the most relevant properties for analyzing the interface deformation and flow dynamics below the interface are the kinematic viscosity and the surface tension. Table 1 shows that the ratio of $v_{\text {slag }} / v_{\text {steel }}$ and $v_{\text {oil }} / v_{\text {water }}$ and also the ratio of surface tensions is matched adequately. 

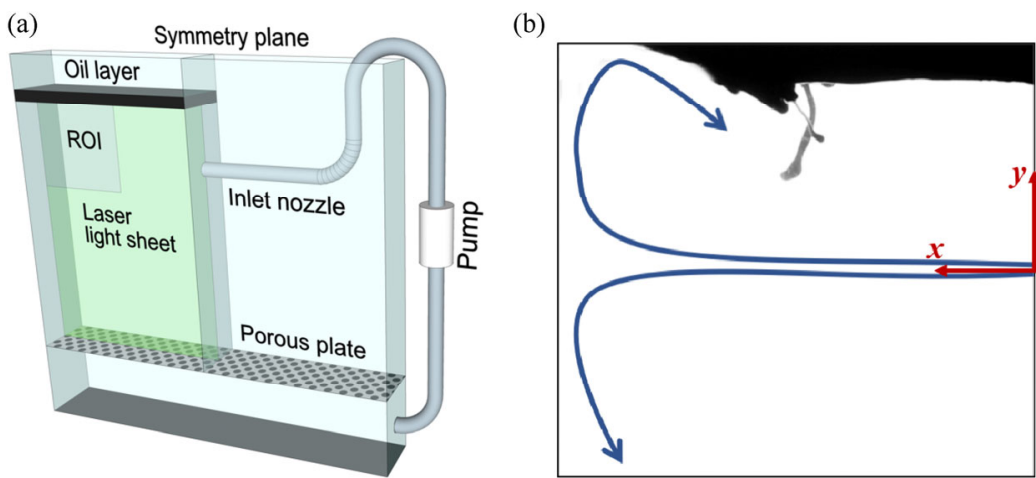

Fig. 2 (a) Setup of the experiment; (b) snapshot taken with LED background illumination.

Table 1 Material properties

\begin{tabular}{lccccc}
\hline \multicolumn{1}{c}{ Material property } & Unit & Steel $\left(1673^{\circ}\right)$ & Slag $\left(1673^{\circ}\right)$ & Water $\left(20^{\circ}\right)$ & Oil $\left(20^{\circ}\right)$ \\
\hline Kinematic viscosity $v$ & $\mathrm{~m}^{2} \cdot \mathrm{s}^{-1}$ & $0.9 \times 10^{-6}$ & $163 \times 10^{-6}$ & $1 \times 10^{-6}$ & $159 \times 10^{-6}$ \\
Density $\varrho$ & $\mathrm{kg} \cdot \mathrm{m}^{-3}$ & 7160 & 2400 & 998 & 863 \\
Surface tension $\sigma$ & $\mathrm{N} \cdot \mathrm{m}^{-1}$ & 1.65 & 0.5 & 0.073 & 0.026 \\
\hline
\end{tabular}

\subsection{PIV and shadowgraphy recordings}

A continuous wave laser with $2.5 \mathrm{~W}$ and $532 \mathrm{~nm}$ wavelength was used to illuminate the center plane of the basin as indicated in Fig. 2. The paraffin oil was colored black by using Sudan black B powder to obtain a good contrast between the main flow and the oil layer. Hollow glass spheres with a mean diameter of $8 \mu \mathrm{m}$ served as tracer particles in the water. The recording of the raw images was done with a Photron Fastcam SA3 running at $1 \mathrm{kHz}$. To obtain a high spatial resolution the region of interest (ROI) was reduced to the upper left corner. The captured area is $125 \mathrm{~mm} \times$ $125 \mathrm{~mm}$ and a $35 \mathrm{~mm}$ F-Mount lens was used on the camera. The CMOS camera chip has a resolution of $1024 \times 1024$ pixels, which leads to an optical resolution of $8.2 \mathrm{px} / \mathrm{mm}$. Comparison with recordings of the complete half of the basin showed, that this reduced ROI significantly improves the image quality and, of course, the quality of the final vector field. Recording the complete basin resulted in peak locking effects which can be avoided by zooming in. A second reason for choosing this ROI was to obtain a spatial resolution similar to the LES study of Saeedipour et al. (2019a). As LES is based on spatial filtering it is not sufficient to obtain a similar temporal resolution in the validation experiments. It is also necessary to provide a similar spatial resolution of the turbulent scales. Thus, experiments of such kind could also be termed scale resolved PIV instead of time-resolved PIV to emphasize that also the important length scales are captured by the experiment.

For both setups, experiments with and without oil layer, ten sets of 1358 images (i.e., the maximum storage capacity of the high-speed camera) have been recorded. Hence, we have an ensemble of ten time-resolved datasets of the mold flow running in steady-state boundary conditions. By a sufficient number of datasets for ensemble averaging we overcome a typical problem of high-speed PIV recordings. Since the available memory of the camera is limited, the observable time interval can become very short when using high frame rates. Thus, while having a good resolution of the unsteady flow, there is a risk of having an insufficient number of frames for statistical evaluations. This can be eliminated by running the experiment several times, which, of course, dramatically increases the processing time.

In addition to the PIV measurements, shadowgraphy recordings were made for the case with the oil layer. A LED panel was used for homogeneous background illumination. These recordings serve to visualize the interface deformation and the occasionally formed ligaments of oil which are entrained into the main flow (c.f. example in Fig. 2(b)). It is important to note that in contrast to the PIV recordings, where a frame rate of $1 \mathrm{kHz}$ is necessary to fully resolve the flow on the time scale, an ordinary video camera running at 25 frames per second is sufficient to completely capture the motion of the water-oil interface. Thus, the dynamics of the liquid-liquid interface is one order of magnitude slower than the turbulent motions in the main flow. Using an ordinary video camera also allows us to capture longer sequences. From these videos the interface motion and frequency of entrainment events can be extracted via digital image processing. Hence, these shadowgraphy recordings provided additional valuable data for the validation of LES results (Saeedipour et al., 2019a). However, in the further discussion in this paper we will focus on the PIV data and the characteristics of the main flow. 


\subsection{Data processing}

Recordings and vector field calculations have been done with the software package Davis 10 from LaVision. To obtain the final vector fields the processing sequence consists of three major steps: (i) perspective correction (ii) automatic masking of the liquid-air and liquid-liquid interfaces (iii) iterative PIV cross-correlation. To account for the large velocity differences in the jet area and the surrounding fluid a pyramid sum of correlation with a pyramid depth of 3 was applied (Sciacchitano et al., 2012). Vector calculation was started at a window size of 96 pixels and iteratively reduced to a final grid size of 24 pixels with an overlap of $75 \%$. The results in a final vector spacing of $0.75 \mathrm{~mm}$. The vector fields have then been exported for further processing and data analysis in Python and Matlab.

The uncertainty of PIV data has been widely discussed in literature over the last three decades. In general, there are two major sources of errors, imperfect tracer particles and inaccurate calculation of the particle displacements. The used tracer particles have a density close to water $\left(\varrho_{\mathrm{p}}=1100 \mathrm{~kg} \cdot \mathrm{m}^{-3}\right)$ which results in a particle relaxation time of $\tau_{\mathrm{p}} \approx 6 \times 10^{-6} \mathrm{~s}$. Hence, we can assume that the Stokes number $S t \ll 1$ and the error due to a slip-velocity of the tracers can be neglected. Most authors report a typical error in the calculated displacement of $1 \%-2 \%$ of the maximum full-scale displacement (Sciacchitano et al., 2013; Westerweel et al., 2013; Wieneke, 2015). This results in a typical particle bias below $0.2 \mathrm{px}$ when the usual quality criteria for PIV measurements are fulfilled (minimum number of particles per interrogation window, maximum particle shift, out-ofplane motion, etc.).

Since deformation of the liquid-liquid interface in the $z$-direction can lead to invisible areas of the interface at the centrally located laser plane, it is important to remember that the automated masking process does not always deliver the precise interface position of the illuminated plane. Instead, it delivers the projection of the interface in the direction of the camera plane. Hence, it is not possible to reliably calculate velocity gradients directly at the interface.

\section{Results and discussion}

\subsection{Instantaneous flow fields}

To give an impression on the overall quality of the results, Fig. 3 shows two instantaneous snapshots for each case with liquid-gas and liquid-liquid interface. The vector field has been coarsened by a factor of four to keep the figure readable. The original data is four times denser which also applies to all other figures with vector field data in this paper. The images give an idea of the highly unsteady nature of the flow fields but also the global flow topology of the clockwise rotating vortex between the jet axis and the interface is visible. The red lines on top give the location of the interface from the automated masking process. The second red line below the interfaces is plotted to mark the layer beneath the interface which was used for data analysis in Sections 3.5 and 3.7. The layer contains 10 vectors and hence covers a region of $7.5 \mathrm{~mm}$ below the interfaces. Calculating averaged or integral values across this layer reduces the problem of having occasional artefacts or spurious vectors in the first data rows beneath the interface because of the shadowing problems that can appear when doing planar PIV below three-dimensionally deformed interfaces.

\subsection{Time-averaged flow fields}

The time-averaged flow fields of the ten different datasets

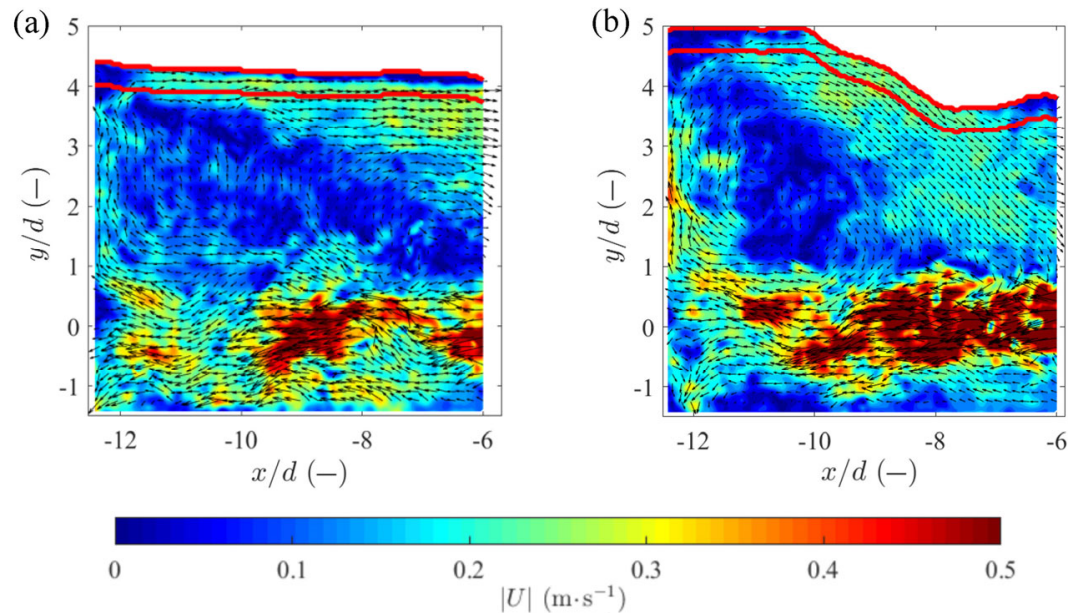

Fig. 3 Instantaneous snapshots for (a) the liquid-gas interface and (b) the liquid-liquid interface. The red lines in (a) and (b) mark the layer beneath the interface which is used for further data analysis in Sections 3.5 and 3.7. The vector fields only show every fourth vector for better readability. 
for both cases presented in Fig. 4 give a good overview of the overall flow situation and the variation between the datasets. The datasets without oil are shown in the left two columns and the results with the oil layer in the right two
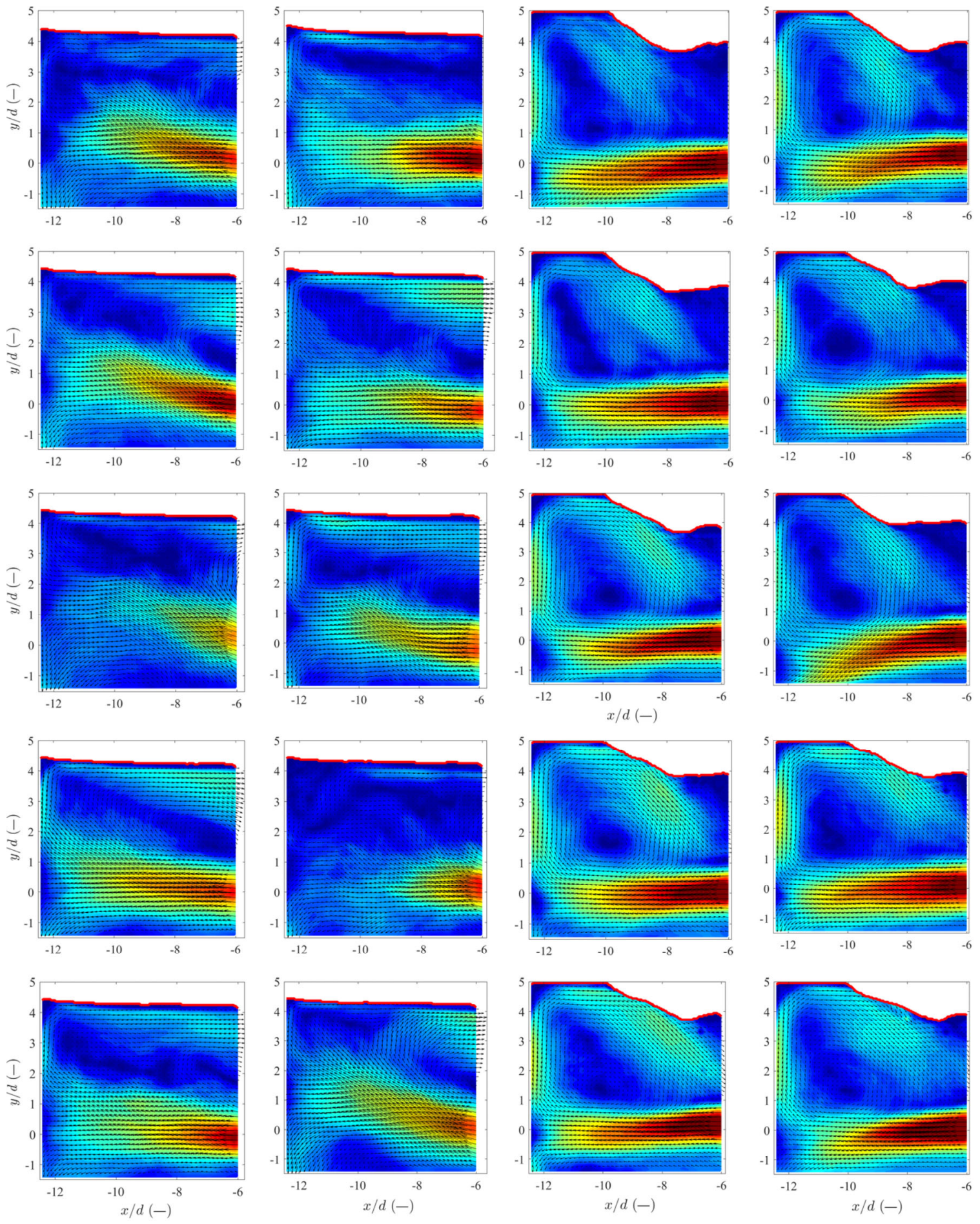

0.1

0.2

0.4

0.5

Fig. 4 Time-averaged flow fields for all 20 datasets. The results for the liquid-gas interface are shown in the left two columns, the flow fields with liquid-liquid interface in the right two columns. 
to the free surface without the presence of an oil layer. For the case with the oil layer the jet either remains horizontal or is slightly deflected downward from the nozzle axis. This is mainly caused by a changing geometry of the clockwise rotating vortex. The liquid-gas interface exhibits a horizontal backflow below the free surface towards the symmetry plane. When an oil layer is present the rotating flow dissipates some of its energy by pushing away the oil layer. This leads to an open area (also commonly called open-eye) where we also have a liquid-gas interface. Due to the deformation of the oil layer, the rotating flow is forced in a downward direction which has a stabilizing effect on the jet itself and causes that deviations of the jet from the horizontal axis are mainly oriented towards negative $y$-coordinates. In general, we can state that the differences between the datasets for the three-phase problem are much smaller than for the two-phase problem.

\subsection{Flow profiles}

The horizontal and vertical flow profiles for various distances to the nozzle outlet are displayed in Figs. 5 and 6. The mean profiles are obtained by time-averaging all ten datasets for the two cases and the velocities are scaled with the jet exit velocity $u_{\text {jet }}$. Around the jet axis the maximum $x$-velocities are about $30 \%$ lower in the two-phase problem compared to the three-phase problem (Fig. 5). These significantly lower mean velocities are caused by the stronger fluctuations of the jet center axis for the liquid-gas interface. The horizontal backflow below the surface is in the similar range for both cases in the open eye area (close to the outer wall) but, of course, vanishes is the area of the entrained oil layer (c.f. the dark blue and red line for $x / d=7$ and 8 in Fig. 5(b)).

For the vertical velocity components given in Fig. 6 we see that the jet beneath the liquid-air interface delivers a lower inertia to the impingement point. Close to the jet axis $(y / d=0.2)$ we already have an upward oriented flow for $x / d<10$, and thus, the vertical velocities close to the wall are much lower. Due to the overall upward deflection of the jet the averaged velocity profile at $y / d=0$ is negative for $x / d>10.5$ since this region is then influenced by the downward deflected part of the jet (c.f. Fig. 4).

In the three-phase setup the profiles are rather similar for all $y / d$-positions. As the main vortex stabilizes the flow we observe negative velocities closer to the nozzle outlet and an upward deflected flow close to the wall.

\subsection{Jet oscillations}

The time-averaged flow fields for the ten datasets in Fig. 4 indicate that the jet center line (position of maximum horizontal velocity) oscillates strongly. Figures $7(\mathrm{a})$ and $7(\mathrm{~b})$
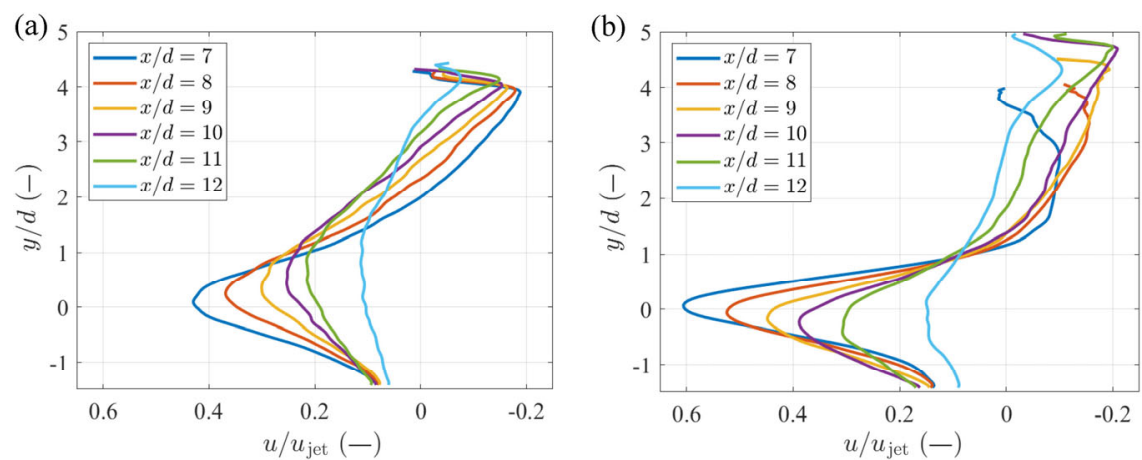

Fig. 5 Time-averaged vertical profiles of $u$-velocities at different distances to the nozzle outlet for (a) the liquid-gas interface and (b) the liquid-liquid interface.
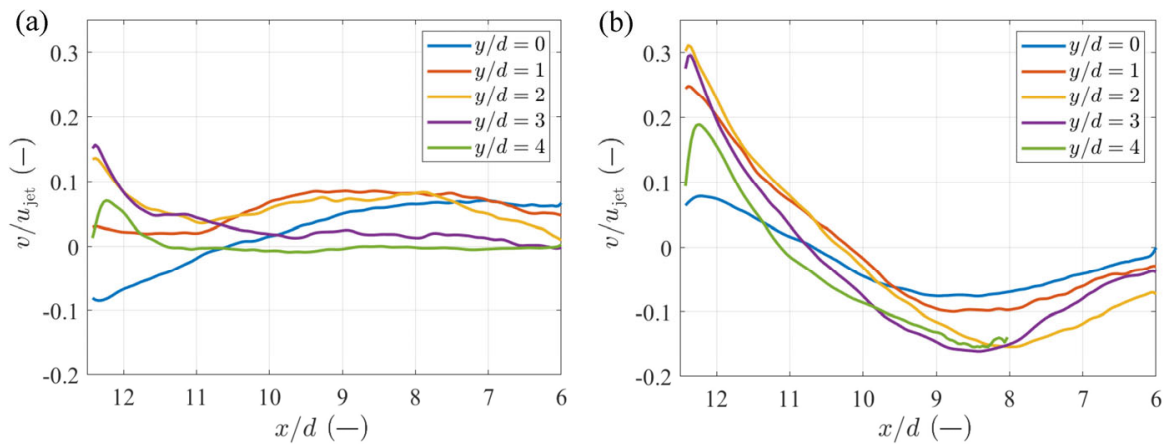

Fig. 6 Time-averaged horizontal profiles of $v$-velocities at different distances to the nozzle outlet for (a) the liquid-gas interface and (b) the liquid-liquid interface. 

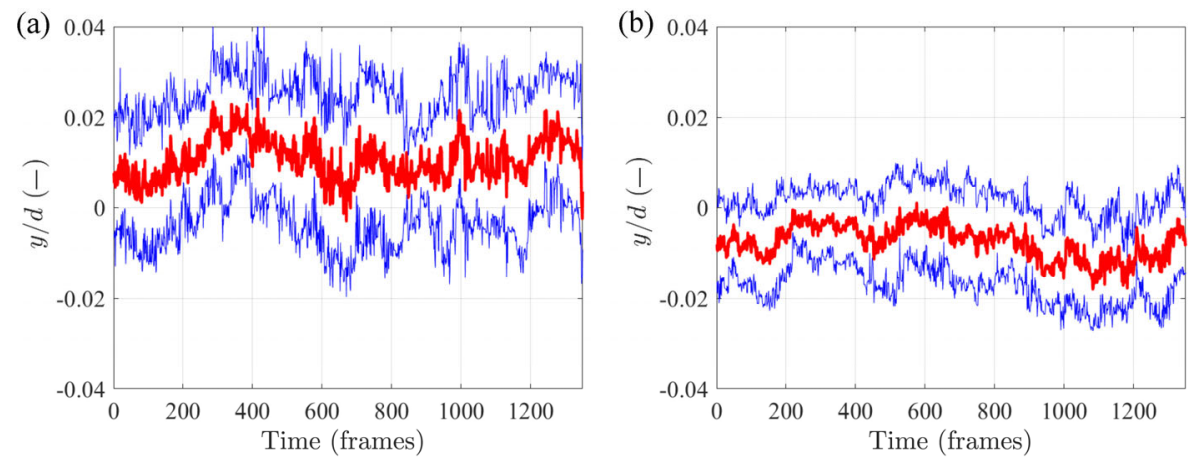

Fig. 7 Temporal evolution of the maximum jet velocity position $u_{\max }$ at $x / d=10$. (a) shows the results for the liquid-gas interface and (b) the liquid-liquid interface. The red lines in (a) and (b) are the ensemble averages, and the blue lines represent the averages \pm the standard deviation.

show the temporal evolution of the maximum $u$-velocity position at a nozzle distance of $x / d=10$. The red lines represent the ensemble averages over the ten datasets and the blue lines indicate the average \pm the standard deviation. We observe that for the liquid-gas case the position of maximum horizontal velocity is shifted upwards from the nozzle center axis and for the liquid-liquid interface it is slightly shifted downwards. The temporal fluctuations are much higher for the jet below a liquid-gas interface. This underlines that the oil layer has a damping effect on the jet oscillations and stabilizes the main flow field.

\subsection{Kinetic energy and turbulent quantities}

When thinking about validating numerical simulations we usually come to the discussion if the kinetic energy and the velocity fluctuations are resolved correctly by the mathematical model. Essentially this can be reduced to the question if the energy distribution between the resolved and unresolved scales is correct and the relevant subgrid terms capture the dominant physical effect correctly.

When we analyze our time-resolved PIV data from that perspective, two interesting quantities are the integral of the kinetic energy

$$
K E=\int_{\Omega} \frac{1}{2} \varrho U^{2} \mathrm{~d} V
$$

as well as the integral of the enstrophy

$$
E=\int_{\Omega} \frac{1}{2}(\nabla \times U)^{2} \mathrm{~d} V
$$

in the proximity of the free surface and liquid-liquid interface. $U$ denotes the velocity vector in Eqs. (1) and (2). The first quantity provides information how much energy is transported to a certain region of the domain, while the latter is a good indicator on the dissipation of turbulence. Figures 8(a) and 8(b) show the kinetic energy and enstrophy integrated for the layer beneath the interface defined in Fig. 3. We clearly see that the integral values of the kinetic energy and enstrophy in this layer are larger for the three-phase problem compared to the two-phase problem. The kinetic energy is increased by $52 \%$ and the enstrophy by $94 \%$. To cross check this results the integration was also done for the entire domain and revealed 39\% increase in kinetic energy and $46 \%$ increase of enstrophy, respectively (results not shown in the figure).

These results disclose some important underlying physics on the effect of the liquid-liquid interface. Although the presence of the oil layer has a stabilizing effect on the jet and strongly dampens its oscillations (Fig. 7), the total kinetic energy and enstrophy in the proximity of the interface is larger than without the oil layer. The increase in both kinetic energy and enstrophy can be explained by two opposing effects in the flow field. On one hand the stabilized main flow leads to higher velocities close to the liquid-liquid interface (c.f. Fig. 4). On the other hand, the liquid-liquid interface can be seen as a flexible wall with a boundary layer of a certain thickness. In the near-interface region, the velocity has to decrease abruptly down to almost zero since the tangential velocity of the interface will be very low. For a rigid wall this would correspond to the no-slip condition. Reducing this higher velocity down to almost zero at the interface induces a strong shear rate in the proximity of the liquid-liquid interface. This high shear region contributes to the production of vorticity, which consequently increases the integral enstrophy.

In addition, Fig. 8(c) shows the root mean squared (RMS) velocity fluctuations:

$$
R M S=\left(\overline{u^{\prime 2}}+\overline{v^{\prime 2}}\right)^{\frac{1}{2}}
$$

across the $x$-coordinate in the interface layer. The RMS values are time-averaged over the entire 10 datasets. The RMS values show that for the liquid-gas interface the velocity 
fluctuations are higher in the upper left corner where the deflected jet hits the free surface. For the liquid-liquid interface we also observe a peak in this region but the clockwise rotating vortex is more stable and a higher percentage of the jet inertia is again deflected and reoriented towards the center plane of the basin. This inertia is pushing against the oil layer and finally leads to a wavy and highly unsteady liquid-liquid interface forming occasional inclusions (c.f. Fig. 2(b)). Beneath this wavy interface the energy is eventually transferred from the main flow to velocity fluctuations which explains the high peak for the liquid-liquid interface in the range $x / d=6.9$ in Fig. $8(\mathrm{c})$.

Figure 9 demonstrates that this energy transfer is not equally distributed across and $u^{\prime}$ and $v^{\prime}$. The figure presents the power spectral densities (PSD) in the interface layer for $u^{\prime}$ and $v^{\prime}$. It is interesting to note that the horizontal velocity fluctuations contain slightly more energy for the liquid-gas interface, but the situation is clearly opposite for the liquid-liquid interface, where the vertical fluctuations contain much more energy. In combination, the results of Figs. 9(a) and 9(b) are in accordance with the findings of Fig. 8(c).

As we track the interface in the automated masking process of the PIV images, we also have the interface position for each snapshot available. Hence, we can also analyze the FFT result of the interface position which is plotted in Fig. 9(c).
The results emphasize the result of Fig. 9(b). Due to the wavy motion of the liquid-liquid interface in the three-phase problem there is much more energy transferred to the vertical velocity fluctuations compared to the two-phase case.

Generally, the energy decay in Figs. 9(a) and 9(b) follows the Kolmogorov $-5 / 3$ law. The little bump around $f=200$ visible for the liquid-gas interface in Figs. 9(b) and 9 (c) is caused by small waves travelling along the interface. Similar waves are also visible in the three-phase setup, but are less pronounced due to the shorter remaining distance of undisturbed liquid-liquid interface (c.f. the upper right region of Fig. 2(b)).

From planar PIV data we generally cannot obtain enough information about the Kolmogorov length scale $\eta_{k}$ since it is not possible to calculate the dissipation rate $\varepsilon$ based on the complete tensor of velocity gradients $\nabla \cdot U$. However, if we follow the linear approximation $\varepsilon=2 \cdot v \cdot E_{\max }$ proposed by Labourasse et al. (2007), where $v$ denotes the kinematic viscosity, we obtain an estimation of the Kolmogorov length scale from the maximum integral enstrophy $E_{\max }$ in the sub-domain. For the values of $E_{\max }$ from Figs. 8(a) and 8(b) the resulting length scales are

$$
\eta_{k}=\left(\frac{v^{3}}{\varepsilon}\right)^{\frac{1}{4}}=\left\{\begin{array}{l}
0.53 m, \text { for the liquid-gas interface } \\
0.46 m, \text { for the liquid-liquid interface }
\end{array}\right.
$$
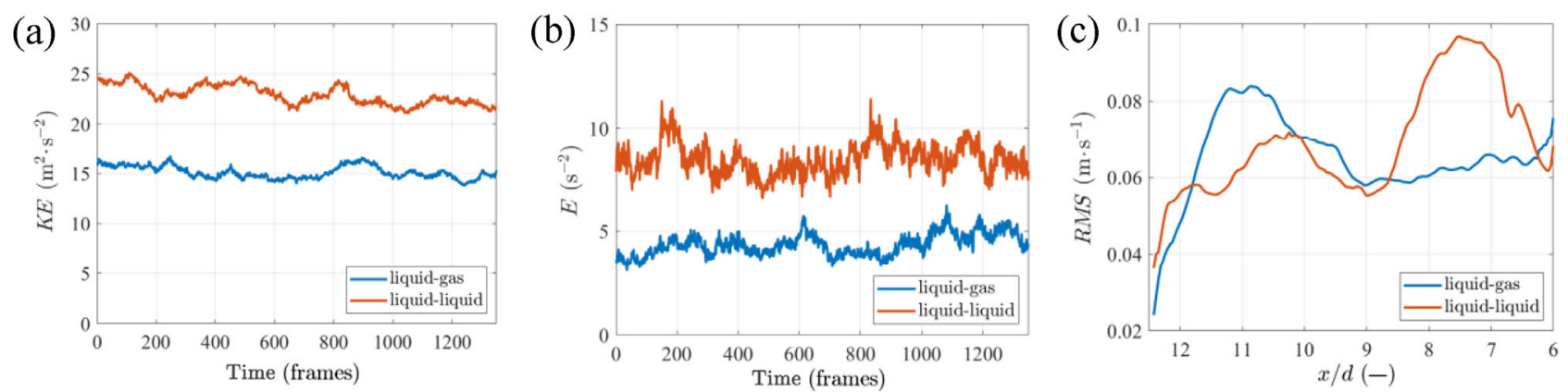

Fig. 8 Integral values of (a) kinetic energy (KE), (b) enstrophy $(E)$, and (c) root mean square velocity fluctuations (RMS) for the defined layer beneath the interfaces.
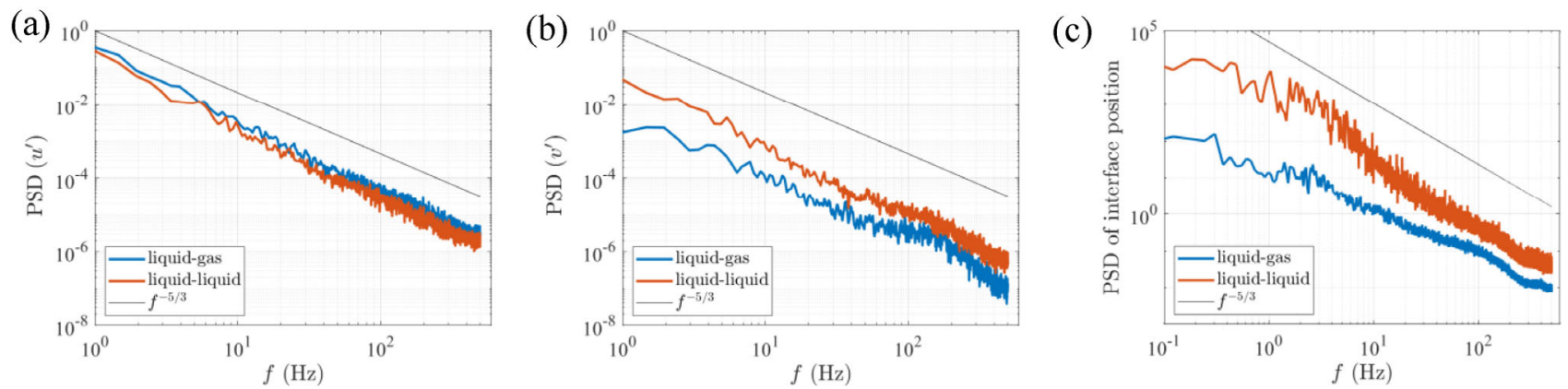

Fig. 9 Power spectral densities of (a) horizontal velocity fluctuations and (b) vertical velocity fluctuations below the liquid-gas and liquid-liquid interfaces calculated for the layer marked in Fig. 3. The black line indicates the $-5 / 3$ decay according to Kolmogorov. (c) shows power spectral density of the interface movement. 
Although the actual Kolmogorov length scale might be smaller, we have a good indication that the resolution of the PIV data is high enough so that the energy spectra in Figs. 9(a) and 9(b) resolve the energy cascade closely down to the Kolmogorov scale.

\subsection{Identification of vortex and strain dominated regions}

The $Q$-criterion is a suitable approach to distinguish between rotation $(Q>0)$ and strain dominated regions $(Q<0)$ in a flow field. For a three-dimensional flow field it is defined by

$$
Q=\frac{1}{2}\left(\|\Omega\|^{2}-\|S\|^{2}\right)
$$

where $S$ and $\Omega$ represent the symmetric and antisymmetric components of the velocity gradient tensor, respectively. From planar PIV data we can only obtain a $2 \mathrm{D}$ velocity gradient tensor and the results are equivalent to other definitions for vortex identification like the $\Delta$ and $\lambda_{2}$ criterion (Jeong and Hussain, 1995). For 2D data Eq. (5) leads to (Chen et al., 2015):

$$
Q=-\frac{1}{2}\left(\frac{\partial u}{\partial x}\right)^{2}-\frac{\partial u}{\partial y} \frac{\partial v}{\partial x}-\frac{1}{2}\left(\frac{\partial v}{\partial y}\right)^{2}
$$

Figure 10 shows the $Q$-criterion results for the time-averaged vector field for one of the datasets for the liquid-gas and liquid-liquid interface. The time-averaged plots give a better insight on the strain and rotation dominated areas instead of showing an instantaneous result, where the results are very weak and might be influenced too much by the abovementioned shadow effect due to the interface deformation. As expected we see that the jet impingement area is strain dominated. Likewise, the layer beneath the liquid-gas and liquid-liquid interfaces is mainly strain dominated. That, of course, does not negate that there might be instantaneous flow situations showing vortex dominated regions close to the liquid-liquid interface. However, the physical mechanism that finally leads to the entrainment of oil into the main liquid is shear.

\subsection{Proper orthogonal decomposition (POD) of vector field time series}

Proper orthogonal decomposition has received a broad acceptance in fluid mechanics to extract important spatial features of a flow field. Taira et al. (2017) presented an overview on POD and related modal decomposition techniques. Essentially, POD is decomposing a dataset into an orthonormal system to capture a maximal amount of energy with a minimal number of eigenmodes. From a fluid mechanics perspective that means the first modes of this orthonormal system will contain the main flow features (large scale vortices), while the higher modes will mainly contain the small-scale turbulent features of the flow. This makes POD an intuitive approach to be used for LES model development and validation, as the LES technique follows a similar approach-the large-scale turbulent motions are resolved by the computational grid, while the small-scale turbulence is captured by the subgrid model. A POD-based comparison between PIV and single-phase LES results for a submerged jet below a wall boundary was presented by Abbasi et al. (2020).

The velocity field as a function of space and time $u\left(r, t_{n}\right)$ can be decomposed to a combination of temporal coefficients $a_{i}\left(t_{n}\right)$ and orthonormal basis functions $\phi_{i}(r)$ :

$$
u\left(r, t_{n}\right)-\langle u\rangle(r)=\sum_{i=1}^{N} a_{i}\left(t_{n}\right) \phi_{i}(r)
$$

The orthonormal basis functions can be found by solving the corresponding eigenvector problem (Sirovich, 1987). To reconstruct the flow field the number of modes $m$ defines the amount of energy reconstructed:

$$
u\left(r, t_{n}\right) \approx\langle u\rangle(r)+\sum_{i=1}^{m} a_{i}\left(t_{n}\right) \phi_{i}(r)
$$

Figure 11 shows two examples of the first modes for the two-phase and the three-phase problems. However, as the
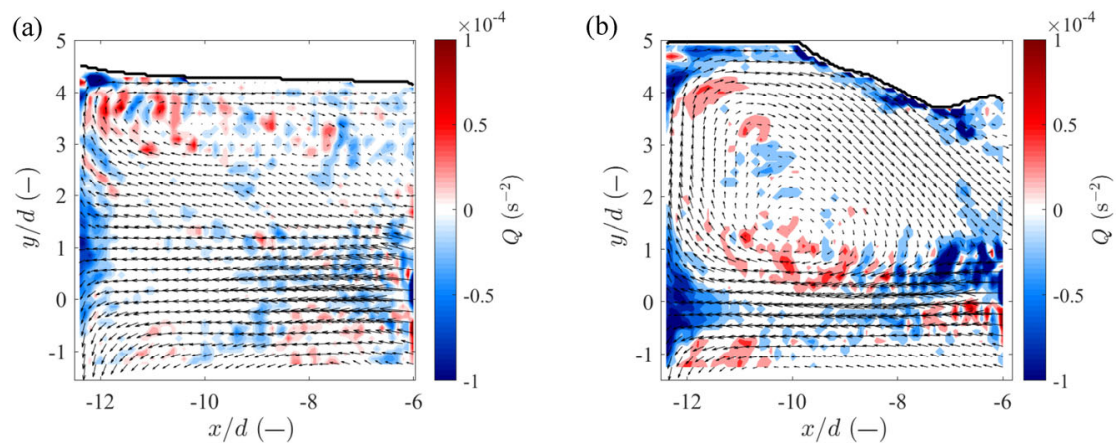

Fig. 10 Q-criteria results for (a) one dataset without liquid-gas and (b) one dataset with liquid-liquid interface. 

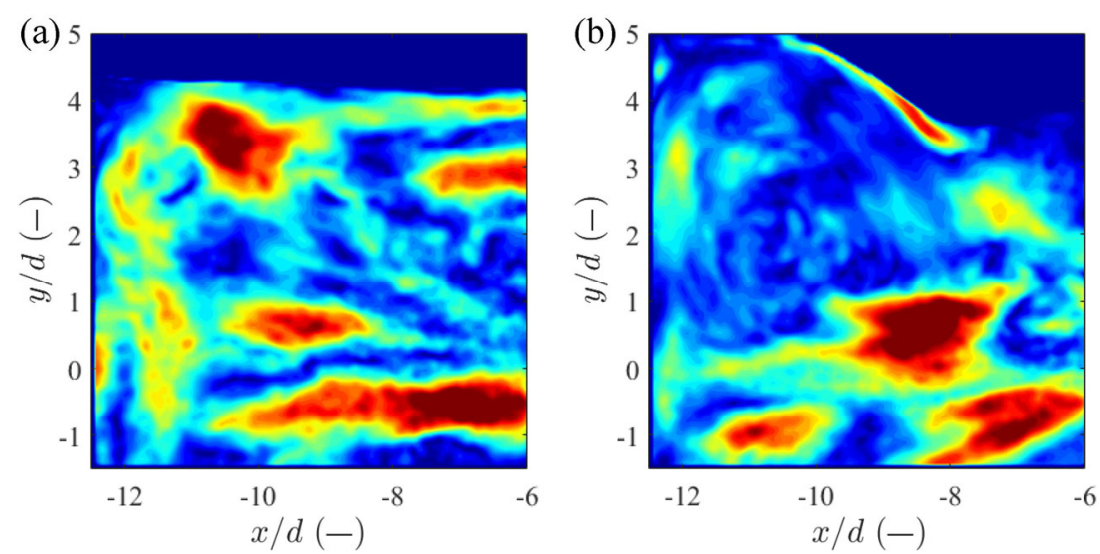

Fig. 11 Exemplary results for mode 1 of the proper orthogonal decomposition for (a) the liquid-gas interface and (b) the liquid-liquid interface.

flow topology between the two cases is very different, it does not make sense to compare the various modes one by one. In the context of LES modelling, it is much more interesting to check the distribution of energy over the mode decomposition. Hence, Fig. 12(a) depicts the cumulative energy distribution of all modes, Fig. 12(b) compares the relative energy content for modes 0 to 4 (where mode 0 is the averaged flow field), and Fig. 12(c) shows the total amount of turbulent kinetic energy captured by reconstructing the flow field with a certain number of modes. The colored bars in Figs. 12(b) and 12(c) are the ensemble-averaged values and the black lines indicate the range of minimum and maximum values over the ten datasets. One can see that mode 0 contains more energy for the liquid-liquid interface as the jet does not oscillate as much as beneath the free surface of the liquid-gas setup. This is also emphasized by the error bars. The range of values for the dataset averages is much larger for the liquid-gas interface than with the additional oil layer. However, modes 1 to 4 show a similar energy content but now the range of the obtained results is larger for the three-phase problem. We can assume that for the case with the oil layer these modes are more affected by the turbulent structures generated by the moving liquid-liquid interface. From Fig. 12(a) we see that the cumulative energy is higher for the first few hundred modes for the liquid-liquid than for the liquid-gas interface, which as well indicates that the amount of energy contained in larger scale fluid motions is higher with the oil layer, while the turbulent kinetic energy is more evenly distributed over the modes without an oil layer. To investigate this argument in detail we once again integrate the turbulent kinetic energy for the layer below the interfaces shown in Fig. 3. Figure 12(c) shows that on average a higher percentage of the turbulent kinetic energy is recovered for the liquid-gas interface when the flow field is reconstructed with less than 100 eigenvectors. This result seems to be contradictory to Fig. 12(a) at the first glimpse, but first, the turbulent kinetic energy does not contain the overall energy of the averaged flow field, and second, this top layer is mainly influenced by small scale motions below the interface. Finally, the result is also in agreement with the power spectral densities discussed in Fig. 9. The overall result of Figs. 9(a) and 9(b) is that the energy content is lower below the liquid-gas interface, thus a lower number of modes is necessary to reconstruct a certain percentage of the turbulent kinetic energy of the original flow field.
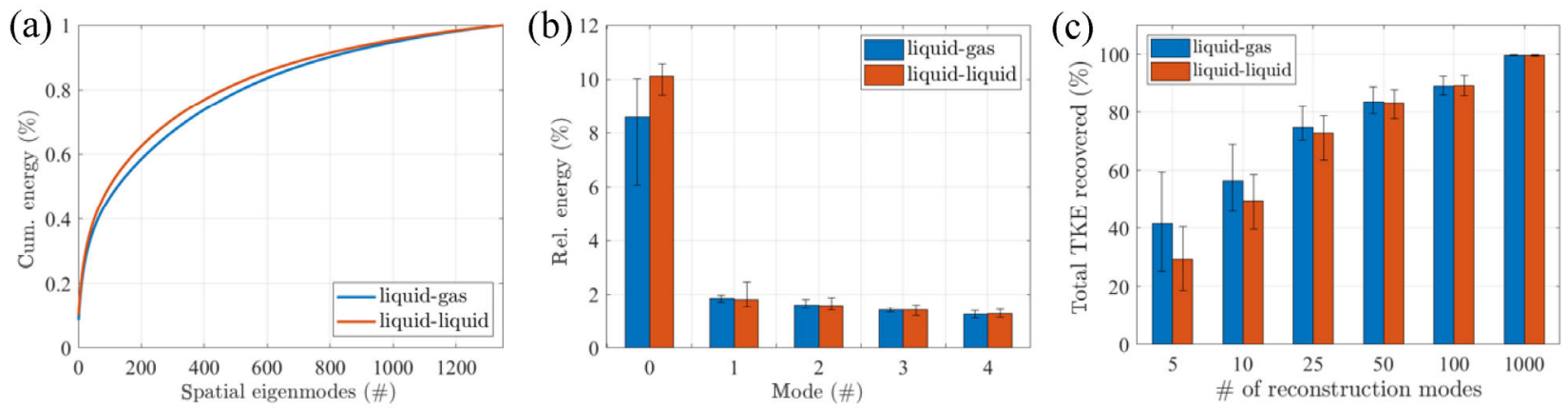

Fig. 12 POD results averaged over all ten datasets for the liquid-gas and liquid-liquid case. (a) Cumulative energy based on the number of eigenmodes. (b) Relative energy content for modes 0 to 4; the black bars indicate the range of results for the ten datasets for both cases. (c) shows the turbulent kinetic energy captured by a certain number of eigenmodes relative to the original flow field. 


\section{Summary and conclusions}

In this paper we discussed the flow topology and turbulent quantities of a confined, deflected submerged jet which is interacting with a liquid-gas (free surface) and a liquid-liquid interface (water plus additional oil layer). In the latter case, the deflected jet is moving part of the oil layer aside and forms an open area (open eye) while entraining the oil layer into the main liquid at the boundary of this open area. Thus, we have a situation of two stratified fluids where the liquid-liquid interface encounters a disturbance caused by large scale motions in the main flow.

Measurements at the very close interface region are not trivial, since besides optical accessibility problems below the three-dimensional interface deformation, it is difficult to provide a sufficient spatial resolution for the calculation of, e.g., interfacial shear stresses. In the present paper, we focused on data analysis of time-resolved planar PIV with high spatial resolution. The following conclusions can be drawn from the presented results:

- Although the data does not allow velocity gradient calculation at the interface, the spatial resolution is high enough to perform a detailed analysis of the flow field and energy content of a small layer below the interface. The PIV data resolves the flow field almost down to the Kolmogorov length scale.

- The results demonstrate that the main flow field is stabilized by the additional oil layer and the oscillations of the jet are highly reduced.

- Due to the stabilized overall flow, more energy is transported towards the interface region by the main vortex which is formed by the jet deflection on the outer wall.

- The interaction of the backflow below the interface and the oil layer leads to a shift of energy from horizontal velocity fluctuations to vertical velocity fluctuations.

- The proper orthogonal decomposition of the flow field demonstrates that the base mode contains a higher amount of energy for the liquid-liquid interface but a larger number of eigenvectors is necessary to reconstruct a certain percentage of the turbulent kinetic energy of the original dataset. Hence, although the oil layer dampens the jet oscillations the turbulent motions are larger below the wavy liquid-liquid interface compared to the free surface of the liquid-gas setup.

It has to be noted that modelling turbulent interfacial flows including the flow situation described in this paper using LES in combination with the VOF method requires further analysis and development of subgrid closure models. This is an ongoing research topic. Thus, high-fidelity data on the turbulence characteristics as well as interfacial flow features are necessary for validation purposes. The presented time-resolved planar PIV experiments, even though with the discussed limitations, could provide an appropriate validation platform for the assessment of numerical simulations. The findings of the present study will have a direct implication in our future subgrid model development for the two-phase LES. This will cover both, functional and structural subgrid models for turbulence-interface interactions based on the VOF method. For future experimental activities, the major goal would be to eliminate the temporarily hidden areas caused by the interface deformation when doing 2D PIV at a $90^{\circ}$ angle. Feasibility tests have shown that the problems with laser reflections that typically occur when using oblique angles for the camera perspective can be reduced when the region of interest is very small. This way the number of lost PIV frames (due to over-saturated areas) is acceptably low. However, by only recording such highly reduced ROIs one would lose the global flow information which would make the interpretation of the near-interface behavior difficult. To solve that issue a multiscale approach using one camera for the global flow field at lower resolution (2D-PIV at $90^{\circ}$ angle) and a second camera with reduced ROI at an oblique angle to keep the region below a deforming liquid-liquid interface always visible would be a feasible approach and part of future research activities.

\section{Acknowledgements}

The author acknowledges the funding support of K1-MET $\mathrm{GmbH}$, metallurgical competence center. The research programme of the K1-MET competence center is supported by COMET (Competence Center for Excellent Technologies), the Austrian programme for competence centers. COMET is funded by the Federal Ministry for Climate Action, Environment, Energy, Mobility, Innovation and Technology, the Federal Ministry for Digital and Economic Affairs, the Federal States of Upper Austria, Tyrol and Styria as well as the Styrian Business Promotion Agency (SFG). Besides the public funding from COMET, this research project is partially financed by the scientific partner Johannes Kepler University Linz and the industrial partner RHI Magnesita.

\section{Funding note}

Open access funding provided by Johannes Kepler University Linz.

\section{Declaration of competing interest}

The authors have no competing interests to declare that are relevant to the content of this article.

\section{References}

Abbasi, S., Puttinger, S., Pirker, S., Lichtenegger, T. 2020. Recurrence 
analysis and time extrapolation of a confined turbulent jet using modal decomposition. Phys Fluids, 32: 075115

Adrian, R. J., Westerweel, J. 2011. Particle Image Velocimetry. Cambridge University Press.

Albrecht, H. E., Borys, M., Damaschke, N., Tropea, C. 2003. Laser Doppler and Phase Doppler Measurement Techniques. Berlin, Heidelberg: Springer Berlin Heidelberg.

Altaharwah, Y. A., Huang, R. F., Hsu, C. M. 2020. Dispersion and upwind-side shear-layer characteristics of forward-inclined transverse jet in crossflow. Exp Therm Fluid Sci, 115: 110104.

André, M. A., Bardet, P. M. 2015. Interfacial shear stress measurement using high spatial resolution multiphase PIV. Exp Fluids, 56: Article Number 132.

Berna, C., Juliá, J. E., Escrivá, A., Muñoz-Cobo, J. L., Pastor, J. V., Micó, C. 2017. Experimental investigation of the entrained droplet velocities in a submerged jet injected into a stagnant water pool. Exp Therm Fluid Sci, 82: 32-41.

Birzer, C. H., Kalt, P. A. M., Nathan, G. J. 2009. The influences of jet precession on near field particle distributions. Int J Multiphase Flow, 35: 288-296.

Birzer, C. H., Kalt, P. A. M., Nathan, G. J. 2011. A method to provide statistical measures of large-scale instantaneous particle clusters from planar images. Exp Fluids, 51: 641-656.

Birzer, C. H., Kalt, P. A. M., Nathan, G. J. 2012. The influences of particle mass loading on mean and instantaneous particle distributions in precessing jet flows. Int J Multiphase Flow, 41: 13-22.

Bruun, H. H. 1995. Hot-Wire Anemometry. Oxford University Press.

Campos, L., Gardin, P., Vincent, S., Caltagirone, J.-P. 2015. Fluid flow description in the vicinity of a liquid steel/slag interface. In: Proceedings of the 6th International Congress on the Science and Technology of Steelmaking.

Chen, Q., Zhong, Q., Qi, M., Wang, X. 2015. Comparison of vortex identification criteria for planar velocity fields in wall turbulence. Phys Fluids, 27: 085101.

Cheng, Y., Torregrosa, M. M., Villegas, A., Diez, F. J. 2011. Time Resolved Scanning PIV measurements at fine scales in a turbulent jet. Int J Heat Fluid Flow, 32: 708-718.

Dong, P., Lu, B., Gong, S., Cheng, D. 2020. Experimental study of submerged gas jets in liquid cross flow. Exp Therm Fluid Sci, 112: 109998.

Fitzgerald, J. A., Garimella, S. V. 1998. A study of the flow field of a confined and submerged impinging jet. Int J Heat Mass Trans, 41: 1025-1034.

Ganapathisubramani, B., Lakshminarasimhan, K., Clemens, N. T. 2008. Investigation of three-dimensional structure of fine scales in a turbulent jet by using cinematographic stereoscopic particle image velocimetry. J Fluid Mech, 598: 141-175.

Ganapathisubramani, B., Lakshminarasimhan, K., Clemens, N. T. 2007. Determination of complete velocity gradient tensor by using cinematographic stereoscopic PIV in a turbulent jet. Exp Fluids, 42: 923-939.

Gebert, B. M., Davidson, M. R., Rudman, M. J. 1998. Computed oscillations of a confined submerged liquid jet. Appl Math Model, 22: 843-850.
Guo, T., Rau, M. J., Vlachos, P. P., Garimella, S. V. 2017. Axisymmetric wall jet development in confined jet impingement. Phys Fluids, 29: 025102.

Harby, K., Chiva, S., Muñoz-Cobo, J. L. 2014. An experimental investigation on the characteristics of submerged horizontal gas jets in liquid ambient. Exp Therm Fluid Sci, 53: 26-39.

Hasslberger, J., Ketterl, S., Klein, M. 2020. A-priori assessment of interfacial sub-grid scale closures in the two-phase flow LES context. Flow Turbul Combust, 105: 359-375.

Hibbeler, L., Thomas, B. 2013. Mold slag entrainment mechanisms in continuous casting molds. Iron Steel Technol, 10: 121-136.

Hirt, C. W., Nichols, B. D. 1981. Volume of fluid (VOF) method for the dynamics of free boundaries. J Comput Phys, 39: 201-225.

Jeong, J., Hussain, F. 1995. On the identification of a vortex. J Fluid Mech, 285: 69.

Ji, C., Li, J., Yang, S., Sun, L. 2013. Large eddy simulation of turbulent fluid flow in liquid metal of continuous casting. J Iron Steel Res Int 20 : 34-39.

Karagozian, A. R. 2014. The jet in crossflow. Phys Fluids, 26: 101303.

Ketterl, S., Klein, M. 2018. A-priori assessment of subgrid scale models for large-eddy simulation of multiphase primary breakup. Comput Fluids, 165: 64-77.

Khan, M. F., Hussain, A., Usmani, A. Y., Yadav, R., Jafri, S. A. H. 2018. Multiphase flow modeling of molten steel and slag flow for different tundish configurations. Mater Today: Proc, 5: 24915-24923.

Klein, M., Ketterl, S., Hasslberger, J. 2019. Large eddy simulation of multiphase flows using the volume of fluid method: Part $1-$ Governing equations and a priori analysis. Exp Comput Multiph Flow, 1: 130-144.

Kwon, S. J., Seo, I. W. 2005. Reynolds number effects on the behavior of a non-buoyant round jet. Exp Fluids, 38: 801-812.

Labourasse, E., Lacanette, D., Toutant, A., Lubin, P., Vincent, S., Lebaigue, O., Caltagirone, J. P., Sagaut, P. 2007. Towards large eddy simulation of isothermal two-phase flows: Governing equations and a priori tests. Int J Multiphase Flow, 33: 1-39.

Lacagnina, G., Romano, G. P. 2015. PIV investigations on optical magnification and small scales in the near-field of an orifice jet. Exp Fluids, 56: Article Number 5.

Larocque, J., Vincent, S., Lacanette, D., Lubin, P., Caltagirone, J.-P. 2010. Parametric study of LES subgrid terms in a turbulent phase separation flow. Int J Heat Fluid Flow, 31: 536-544.

Lawson, N. J., Arruda, M. P., Davidson, M. R. 2005. Control of a submerged jet in a thin rectangular cavity. J Fluids Struct, 20: 1025-1042.

Lawson, N. J., Davidson, M. R. 2001. Self-sustained oscillation of a submerged jet in a thin rectangular cavity. J Fluids Struct, 15: 59-81.

Liu, Z., Li, B. 2018. Effect of vertical length on asymmetric flow and inclusion transport in vertical-bending continuous caster. Powder Technol, 323: 403-415.

Liu, Z., Qi, F., Li, B., Jiang, M. 2014. Vortex flow pattern in a slab continuous casting mold with argon gas injection. J Iron Steel Res Int, 21: 1081-1089.

Ma, J., Song, Y., Zhou, P., Cheng, W., Chu, S. 2018. A mathematical approach to submerged horizontal buoyant jet trajectory and a criterion for jet flow patterns. Exp Therm Fluid Sci, 92: 409-419. 
Meyer, K. E., Pedersen, J. M., Özcan, O. 2007. A turbulent jet in crossflow analysed with proper orthogonal decomposition. J Fluid Mech, 583: 199-227.

Pirker, S. 2010. Towards efficient modelling of slag entrainment during metallurgical processes. Steel Res Int, 81: 623-629.

Puttinger, S., Holzinger, G., Pirker, S. 2013. Investigation of highly laden particle jet dispersion by the use of a high-speed camera and parameter-independent image analysis. Powder Technol, 234: 46-57.

Raffel, M., Willert, C. E., Wereley, S. T., Kompenhans, J. 2007. Particle Image Velocimetry. Berlin, Heidelberg: Springer Berlin Heidelberg.

Ramos-Banderas, A,. Morales, R. D., Sánchez-Pérez, R., GarcíaDemedices, L., Solorio-Diaz, G. 2005. Dynamics of two-phase downwards flows in submerged entry nozzles and its influence on the two-phase flow in the mold. Int J Multiphase Flow, 31: 643-665.

Ren, L., Ren, Y., Zhang, L., Yang, J. 2019. Investigation on fluid flow inside a continuous slab casting mold using particle image velocimetry. Steel Res Int, 90: 1900209.

Saeedipour, M., Puttinger, S., Doppelhammer, N., Pirker, S. 2019a. Investigation on turbulence in the vicinity of liquid-liquid interfaces - Large eddy simulation and PIV experiment. Chem Eng Sci, 198: 98-107.

Saeedipour, M., Schneiderbauer, S. 2019. A new approach to include surface tension in the subgrid eddy viscosity for the two-phase LES. Int J Multiphase Flow, 121: 103128.

Saeedipour, M., Vincent, S., Pirker, S. 2019b. Large eddy simulation of turbulent interfacial flows using Approximate Deconvolution Model. Int J Multiphase Flow, 112: 286-299.

Sciacchitano, A., Scarano, F., Wieneke, B. 2012. Multi-frame pyramid correlation for time-resolved PIV. Exp Fluids, 53: 1087-1105.

Sciacchitano, A., Wieneke, B., Scarano, F. 2013. PIV uncertainty quantification by image matching. Meas Sci Technol, 24: 045302.

Semeraro, O., Bellani, G., Lundell, F. 2012. Analysis of time-resolved PIV measurements of a confined turbulent jet using POD and Koopman modes. Exp Fluids, 53: 1203-1220.

Sirovich, L. 1987. Turbulence and the dynamics of coherent structures. I. Coherent structures. Quart Appl Math, 45: 561-571.

Srinivas, P. S., Mishra, D. K., Gupta, R., Korath, J. M., Jana, A. K. 2019. Vortex characteristics due to nozzle clogging in water caster mould: Modelling and validation. Can Metall Quart, 58: 308-324.

Stolz, S., Adams, N. A. 1999. An approximate deconvolution procedure for large-eddy simulation. Phys Fluids, 11: 1699-1701.

Taira, K., Brunton, S. L., Dawson, S. T. M., Rowley, C. W., Colonius, T., McKeon, B. J., Schmidt, O. T., Gordeyev, S., Theofilis, V., Ukeiley, L. S. 2017. Modal analysis of fluid flows: An overview. AIAA J, 55: 4013-4041.

Vakhrushev, A., Wu, M., Ludwig, A., Tang, Y., Nitzl, G., Hackl, G. 2014. Experimental verification of the 3-phase continuous casting simulation using a water model. In: Proceedings of the 8 th European Continuous Casting Conference.
Vincent, S., Tavares, M., Fleau, S., Mimouni, S., Ould-Rouiss, M., Estivalezes, J.-L. 2018. A priori filtering and LES modeling of turbulent two-phase flows application to phase separation. Comput Fluids, 176: 245-259.

Weiland, C., Vlachos, P. P. 2013. Round gas jets submerged in water. Int J Multiphase Flow, 48: 46-57.

Wen, Q. Kim, H. D., Liu, Y. Z., Kim, K. C. 2014. Dynamic structures of a submerged jet interacting with a free surface. Exp Therm Fluid Sci, 57: 396-406.

Westerweel, J., Elsinga, G. E., Adrian, R. J. 2013. Particle image velocimetry for complex and turbulent flows. Ann Rev Fluid Mech, 45: 409-436.

Westerweel, J., Fukushima, C., Pedersen, J. M., Hunt, J. C. R. 2009. Momentum and scalar transport at the turbulent/non-turbulent interface of a jet. J Fluid Mech, 631: 199-230.

Wieneke, B. 2015. PIV uncertainty quantification from correlation statistics. Meas Sci Technol, 26: 074002.

Wygnanski, I., Fiedler, H. 1969. Some measurements in the selfpreserving jet. J Fluid Mech, 38: 577-612.

Yokoya, S., Takagi, S., Iguchi, M., Marukawa, K., Hara, S. 2000. Swirling flow effect in immersion nozzle on flow in slab continuous casting mold. ISIJ Int, 40: 578-583.

Yu, H., Zhu, M., Wang, J. 2010. Interfacial fluctuation behavior of steel/slag in medium-thin slab continuous casting mold with argon gas injection. J Iron Steel Res Int, 17: 5-11.

Zhang, X., Shen, H. 2006. Lattice BGK model simulation of asymmetric flow inside a continuous slab casting mold. J Hydrodyn, 18: 431-435.

Zhang, X., Zhang, W., Jin, J., Evans, J. W. 2007. Flow of steel in mold region during continuous casting. J Iron Steel Res Int, 14: 30-35.

Zhao, P., Li, Q., Kuang, S. B., Zou, Z. 2017. Mathematical modeling of liquid slag layer fluctuation and slag droplets entrainment in a continuous casting mold based on VOF-LES method. High Temp Mater Process, 36: 551-565.

Zhao, P., Zhou, L. 2019. Mathematical modelling of slag entrainment and entrained droplets in a continuous casting mould. Ironmak Steelmak, 46: 886-895.

Open Access This article is licensed under a Creative Commons Attribution 4.0 International License, which permits use, sharing, adaptation, distribution and reproduction in any medium or format, as long as you give appropriate credit to the original author(s) and the source, provide a link to the Creative Commons licence, and indicate if changes were made.

The images or other third party material in this article are included in the article's Creative Commons licence, unless indicated otherwise in a credit line to the material. If material is not included in the article's Creative Commons licence and your intended use is not permitted by statutory regulation or exceeds the permitted use, you will need to obtain permission directly from the copyright holder.

To view a copy of this licence, visit http://creativecommons.org/licenses/by/4.0/. 